\title{
Níveis de lactecidemia durante jogo de pólo aquático: estudo preliminar
}

\author{
Emilson Colantonio ${ }^{1,2,3}$, Emerson Franchini ${ }^{1}$, \\ Karin Ayumi Matsushigue ${ }^{1}$ e Maria Augusta Peduti Dal Molin Kiss ${ }^{1}$
}

\section{RESUMO}

O jogo de pólo aquático é uma modalidade de característica intermitente, disputada em quatro períodos de $7 \mathrm{~min}$ com $2 \mathrm{~min}$ de intervalo entre estes. Em caso de prorrogação, há um período de repouso de 5 min e, a seguir, serão jogados dois períodos de 3 min cada, com um intervalo de 1 min para as equipes trocarem de lado. Para modalidades com estas características, tem sido sugerida a utilização da mensuração de lactato sanguíneo como indicador da solicitação metabólica. Em função disso, o presente estudo tem como objetivo analisar a concentração de lactato sanguíneo [La] imediatamente após cada um dos períodos de um jogo do Campeonato Paulista Infanto-Juvenil de 1999 (disputa de 3- lugar) e verificar se ocorre diferença na [La] entre os períodos e a prorrogação. Foram sujeitos deste estudo cinco atletas da equipe de nível estadual do Clube Internacional de Regatas (idade $=15,8 \pm 0,4$ anos; massa corporal $=71,5 \pm 11,0 \mathrm{~kg}$ e estatura $=180 \pm 4 \mathrm{~cm}$ ). O sangue foi coletado no lóbulo da orelha e analisado pelo lactímetro Yellow Springs modelo 1500 Sport. Para a comparação da [La] entre os períodos foi utilizada a estatística não paramétrica de Kruskal-Wallis Anova. Os resultados da [La] não diferiram (qui-quadrado $=0,9615 ; \mathrm{gl}=4 ; \mathrm{p}=0,9156$ ) entre os períodos (período $1=4,64 \pm 1,21 \mathrm{mM}$; período $2=$

1. Laboratório de Desempenho Esportivo/Cenesp/EEFEUSP.

2. Unicid.

3. Unimonte.

Recebido em: 28/7/2001

Aceito em: 25/9/2001.

Endereço para correspondência:

Prof. Ms. Emilson Colantonio

Ladesp/Cenesp/Escola de Educação Física e Esporte da Universidade de São Paulo

Av. Mello Moraes, 65 - Butantã

05508-900 - São Paulo, SP

E-mail: nunoec@uol.com.br
$5,08 \pm 1,95 \mathrm{mM}$; período $3=4,72 \pm 1,33 \mathrm{mM}$; período $4=$ $4,32 \pm 1,12 \mathrm{mM}$; prorrogação $=4,11 \pm 1,03 \mathrm{mM}$ ). Os resultados indicam que a contribuição da glicólise anaeróbia não foi predominante, sugerindo haver solicitação do sistema anaeróbio aláctico (ATP-CP) nos momentos decisivos do jogo e do metabolismo aeróbio no intervalo entre as ações. Devido a carência de estudos dessa natureza relacionados a essa modalidade esportiva, é oportuna a elaboração de trabalhos subseqüentes que possam trazer informações mais conclusivas.

Palavras-chave: Lactato. Pólo aquático. Situação de jogo.

\section{ABSTRACT}

\section{Lactacidemic levels during water polo game: preliminary study}

The water polo game is an sport event of intermittent characteristic, with four periods of 7 min and 2 min of interval between the periods. In cases of game extension, after a 5-min resting period, the game follows on with two game periods of 3 min each, with a 1-minute interval for the teams to change sides. In events with these characteristics, the use of blood lactate measurement has been suggested as an indicative of metabolic response. For this reason, the aim of the present study was to check blood lactate accumulation [La] immediately after each period of a game of the 1999 State Championship for Children and Adolescents (a game to classify the $3^{\text {rd }}$ place in the Championship), and to verify if there are [La] differences between game periods and extension periods. The sample was made of five athletes of the Clube Internacional de Regatas Team (age $=15.8 \pm 0.4$ years; body mass $=71.5 \pm$ $11.0 \mathrm{~kg}$ and height $=180 \pm 4 \mathrm{~cm}$ ). Blood was drawn from the ear lobe and analyzed by a Yellow Springs model 1500 Sport analyzer. Kruskal-Wallis Oneway statistical analysis was used to compare [La] among the periods. The results of the [La] are not different (chi-square $=0.9615 ; \mathrm{p}$ 
$=0.9156$ ), in the different periods (period $1=4.64 \pm 1.21$ $\mathrm{mM} ;$ period $2=5.08 \pm 1.95 \mathrm{mM} ;$ period $3=4.72 \pm 1.33$ $\mathrm{mM} ;$ period $4=4.32 \pm 1.12 \mathrm{mM} ;$ extension $=4.11 \pm 1.03$ $m M)$. Results indicate low demand from the anaerobic glycolysis, and suggest that there is a higher demand from the anaerobic system (ATP-CP) in the decisive moments of the game, and from the aerobic metabolism in the interval between the periods. Due to the lack of studies involving water polo, the preparation of new papers that bring more conclusive information may be appropriate.

Key words: Lactate. Water polo. Game situation.

\section{INTRODUÇÃO}

A modalidade esportiva pólo aquático (PA) vem sendo praticada desde o final do século passado. Os ingleses praticavam esse esporte sobre barris de madeira e basicamente a troca de passes era realizada sem muita movimentação, diferentemente dos dias de hoje. A Europa é o berço desse esporte, sendo a Hungria o país que mais conquistou títulos internacionais, incluindo 11 medalhas olímpicas. O PA e o futebol são as mais antigas modalidades olímpicas por equipe, participando nos Jogos Olímpicos de Paris em 1900.

O jogo possui característica intermitente e é disputado em quatro períodos de $7 \mathrm{~min}$ com $2 \mathrm{~min}$ de intervalo. Em caso de prorrogação, há um período de repouso de 5 min e, a seguir, serão jogados dois períodos de $3 \mathrm{~min}$ cada com um intervalo de $1 \mathrm{~min}$ para as equipes trocarem de lado. Persistindo a situação de empate, haverá um período de 1 min e um terceiro período de jogo começará terminando assim que o primeiro gol for assinalado (golden goal).

A duração total de um jogo está por volta de 55 a $60 \mathrm{~min}$. Quando há prorrogação pode-se alcançar mais de 70min de jogo, pois em todas as paralisações a marcação do tempo deverá parar até que a bola seja colocada novamente em jogo, seja deixando a mão do jogador cobrando o tiro apropriado, seja tocada por um jogador em seguida a um tiro neutro. Em média cada período dura cerca de $12 \mathrm{~min}$. As equipes são formadas por 13 jogadores, sete atuam na água, sendo seis na linha e um goleiro.

Análises de jogo têm demonstrado que o PA é um esporte intermitente composto de atividades curtas e intensas de menos de 15 segundos de duração mescladas com intervalos de atividades de baixa intensidade que em média duram menos de 20 segundos $^{1}$. Para modalidades com estas características, tem sido sugerida a utilização da mensuração do lactato sanguíneo como indicador da solicitação metabólica ${ }^{2}$. As medidas da [La] podem trazer informações sobre o grau de contribuição do sistema láctico e a caracterização do perfil metabólico da atividade motora. A partir desse conhecimento, a prescrição do treinamento se torna mais específica e direcionada ao trabalho adequado para os sistemas predominantes.

Apesar do PA ser praticado há muitos anos, existe uma carência de estudos sobre essa modalidade na literatura internacional. Em termos de literatura nacional, esse fato acentua-se ainda mais. Sendo assim, o presente estudo tem como objetivo analisar a concentração de lactato sanguíneo [La] imediatamente após cada um dos períodos de um jogo do Campeonato Paulista Infanto-Juvenil de 1999 (disputa de 3o lugar), e verificar se houve diferença na [La] entre os períodos e a prorrogação.

\section{MÉTODOS}

Foram sujeitos deste estudo cinco atletas do sexo masculino da equipe do Clube Internacional de Regatas, sendo que todos os jogadores de linha tinham pelo menos três anos de prática esportiva sistemática e treinamento. Previamente, um termo de consentimento informado foi fornecido aos atletas para leitura, ciência e aprovação das avaliações a serem realizadas. As características gerais da amostra encontram-se na tabela 1.

As amostras de sangue foram coletadas no lóbulo da orelha dos avaliados logo após o término de cada período, durante os intervalos entre os quartos da partida, de forma a interferir o menos possível na dinâmica do jogo, bem como nas intervenções feitas pelos treinadores. Cada avaliador coletou amostras de sangue de apenas um atleta. Devido à dificuldade de coleta, algumas foram perdidas por motivos metodológicos, diminuindo, então, o número de amostras.

A análise do sangue coletado por capilar foi realizada através da utilização do lactímetro Yellow Springs modelo 1500 Sport. Foram coletadas amostras de $25 \mu \mathrm{l}$ e armazenados com $50 \mu \mathrm{l}$ de fluoreto de sódio a $1 \%$.

Devido à situação de jogo em um campeonato (disputa de 3 o lugar), houve uma necessidade imperativa de assepsia prévia à coleta no lóbulo da orelha.

TABELA 1

Valores de média (x) e desvio padrão (sd) para idade, massa corporal e estatura dos atletas estudados

$\mathbf{x}$

sd

Idade (anos)

Massa corporal $(\mathrm{kg})$

15,80

71,50

Estatura $(\mathrm{cm})$
0,40

11,00

4,00 
Para comparação da [La] entre os períodos foi utilizada a estatística não paramétrica de Kruskal-Wallis Anova.

\section{RESULTADOS}

Os resultados da [La] não diferiram (qui-quadrado = $0,9615 ; \mathrm{gl}=4 ; \mathrm{p}=0,9156)$ entre os períodos de jogo. Os valores médios de [La] em $\mathrm{mmol} / \mathrm{l}(\mathrm{mM})$ e seus respectivos desvios padrões entre cada período e após a prorrogação podem ser vistos na tabela 2 .

\section{DISCUSSÃO}

Individualmente, os valores de [La] dos atletas mostraram-se com uma certa estabilidade, alternando em poucas oportunidades entre um período e outro do jogo.

Vale ressaltar os valores de [La] do atleta 4 (A4), cuja função primária nessa modalidade é fazer gol, portanto, é aquele atacante que joga quase que exclusivamente de costas para o gol adversário, com pouco deslocamento. Os valores de [La] do A4 mostraram-se abaixo dos demais durante todo o jogo, não chegando a ultrapassar os $3 \mathrm{mM}$. Neste caso, parece que suas ações durante o jogo aconteceram basicamente em função do sistema anaeróbio aláctico nos momentos decisivos e com a participação do metabolismo aeróbio durante os intervalos dessas ações. Diferentemente dos demais, que, de maneira geral, mantiveram-se próximos aos níveis de intensidades compatíveis ao limiar anaeróbio.

Apesar de um grande número de publicações sobre aspectos técnicos e táticos do PA, pesquisas na literatura sobre o tema em questão são limitadas. Existem alguns estudos enfocando as características morfológicas dos jogadores $^{3-5}$; aspectos de arrasto e propulsão durante as atividades do $\mathrm{PA}^{6}$; características das lesões habituais no $\mathrm{PA}^{7}$, eletromiografia de músculos associados ao arremesso da bola no $\mathrm{PA}^{8}$ e a biomecânica do batimento de perna do PA, nado crawl e técnicas de nadar com a cabeça para fora ${ }^{9,10}$.

\section{TABELA 2}

Valores de concentração de lactato (mM) e seus correspondentes desvios padrões entre os períodos de jogo e após a prorrogação

\begin{tabular}{lccccc}
\hline Atletas & $\mathbf{P 1}$ & $\mathbf{P 2}$ & $\mathbf{P 3}$ & $\mathbf{P 4}$ & Prorrogação \\
A1 & 6,09 & 6,88 & 5,88 & 5,82 & 3,12 \\
A2 & 5,59 & 5,24 & 5,56 & 4,47 & 5,21 \\
A3 & 4,53 & 3,32 & 5,82 & 3,76 & 5,24 \\
A4 & 2,59 & 2,47 & 2,56 & 2,53 & 2,76 \\
A5 & 4,38 & 7,50 & 3,76 & 5,03 & 4,21 \\
Média & 4,64 & 5,08 & 4,72 & 4,32 & 4,11 \\
sd & 1,21 & 1,95 & 1,33 & 1,12 & 1,03 \\
\hline
\end{tabular}

Estudos mais recentes têm investigado as características fisiológicas dos jogadores de PA; a demanda durante os jogos; as práticas de treinamento e periodização do treinamento de PA, buscando novas evoluções ${ }^{11-14}$. Avaliações fisiológicas obtidas durante o jogo indicam um efeito acumulativo das repetidas seqüências de atividades e sugerem que existe uma alta demanda metabólica dos atletas ${ }^{1}$. Em termos gerais, as atividades físicas realizadas durante o jogo de PA são inúmeras e podem ser classificadas de acordo com as habilidades envolvidas, as regras que delineiam essas atividades e a velocidade dos movimentos ${ }^{11}$. Especificamente, quanto à intensidade, duração e freqüência das múltiplas atividades realizadas pelos jogadores durante uma partida de PA, sabe-se que as atividades duram até $20 \mathrm{seg}$ com movimentos intensos e "sprints" que levam, em média, de 7 a 14seg. Pela brevidade de tais atividades, o metabolismo anaeróbio e a potência muscular são altamente dependentes. Entretanto, essas atividades são realizadas em seqüências resultando em um efeito acumulativo, portanto de longa duração, com períodos de alta e moderada intensidades que constituem, aproximadamente, dois terços do tempo total de jogo.

A complexidade, variação e natureza intermitente do PA faz com que a avaliação e interpretação das respostas fisiológicas atuais relacionadas ao treinamento e competição sejam tecnicamente difíceis.

Os valores médios de [La] do presente estudo foram para $\mathrm{P} 1=4,64 \pm 1,21 ; \mathrm{P} 2=5,08 \pm 1,95 ; \mathrm{P} 3=4,72 \pm 1,33 ; \mathrm{P} 4=$ $4,32 \pm 1,12$ e a prorrogação $=4,11 \pm 1,03$. Os valores médios de [La] encontrados para a amostra em questão sugerem que durante os períodos de jogo os atletas mantiveram-se próximos aos níveis de [La] correspondentes ao limiar anaeróbio, isto é, limiar de lactato de $4 \mathrm{mM}$.

Esses valores mostraram-se similares aos encontrados por Konstantaki et al. ${ }^{15}$, os quais investigaram oito jogadoras de PA (idade $=26,7 \pm 5,7$ anos, massa corporal $=65,3 \pm$ $7,0 \mathrm{~kg}$ e estatura $=169 \pm 6 \mathrm{~cm})$ em situação de jogo $(\mathrm{P} 1=$ $3,5 \pm 0,4 \mathrm{mM} ; \mathrm{P} 2=4,3 \pm 0,5 \mathrm{mM} ; \mathrm{P} 3=4,3 \pm 0,7 \mathrm{mM}$ e $\mathrm{P} 4=$ $4,6 \pm 0,5 \mathrm{mM})$.

Hollander et al ${ }^{16}$ encontraram valores individuais de [La] em jogadoras alemãs de nível nacional na faixa de 2 a $10 \mathrm{mM}$ com valores médios de $5 \mathrm{mM}$, similares aos valores encontrados por Konstantaki et al. ${ }^{15}$ e o presente estudo. Infelizmente, a literatura internacional não conta com estudos investigando jogadores(as) adolescentes para possíveis comparações de aspectos metabólicos entre gêneros e idade. Sabe-se que os níveis de [La] são mais baixos em crianças do que em adultos a uma mesma intensidade relativa de exercício ${ }^{17-19}$.

Pinnington et al. ${ }^{11}$, investigaram a freqüência cardíaca em combinação com valores de [La] durante jogos de PA e 
observaram moderada demanda do sistema glicolítico, de acordo com o estudo de Hollander et al. ${ }^{16}$. No estudo de Pinnington et al. ${ }^{11}$, registrou-se a FC através de telemetria e o valor mais alto encontrado foi de $183 \mathrm{bpm}$. Durante os períodos de jogo, a FC dos jogadores esteve por volta de $82,80 \%$ em relação ao valor médio da FC máxima do grupo. Hollander et al. ${ }^{16}$, encontraram um valor médio de FC, obtida através de teste, de 189,20 \pm 9,30bpm. Porém, durante o jogo, o valor médio foi de 185,00 $\pm 3,73 \mathrm{bpm}$ e valores de [La] entre 1,52 e 9,79mM com um valor médio de $5,34 \pm 2,11 \mathrm{mM}$.

Observações de jogadores durante competições internacionais mostraram que em aproximadamente $85 \%$ do tempo as velocidades de movimento no plano horizontal foram abaixo daquelas durante a velocidade constante de nado livre, levando a níveis de lactato menores ou iguais a $2 \mathrm{mM}^{13}$, ${ }^{14,20}$. Esses dados têm sido interpretados como um reflexo de uma alta demanda do sistema anaeróbio aláctico, uma alta demanda do sistema aeróbio para a ressíntese da creatina fostato e menor ênfase no metabolismo anaeróbio láctico para o fornecimento de energia ${ }^{1}$.

Coletas de sangue para análise das [La] realizadas durante o jogo e após os períodos de jogo, em jogadores de elite espanhóis e italianos, mostraram valores individuais de 2 a $12 \mathrm{mM}$ apresentando valores médios de 7 a $9 \mathrm{mM}^{12,21}$, evidenciando uma maior participação do sistema glicolítico em atletas adultos de alto nível do sexo masculino.

\section{REFERÊNCIAS}

1. Smith HK. Applied physiology of water polo. Sports Med 1998;26:31734.

2. Jacobs I. Blood lactate: implications for training and sports performance. Sports Med 1986;3:10-25.

3. Hebbelinck M, Carter JEL, De Garay A. Body build and somatotype of Olympic swimmers, divers and water polo players. In: Clarys JP, Lewile L, editors. Swimming II. Baltimore (MD): University Park Press, 1975: 285-305.

4. Vertommen L, Clarys JP, Welch W. Body measurements and heart morphology of water polo players. In: Swimming III - International Series on Sport Sciences. Proceedings of the Third International Symposium of Biomechanics in Swimming. University of Alberta, Edmonton, Canada, 1979;307-19.

5. Carter JEL, Ackland TR. Kinanthropometry in aquatic sports - A study of world class athletes. Human Kinetics, Champaign, Il., 1994.

6. Clarys JP. Human morphology and hydrodynamics. In: Swimming III. Baltimore (MD): University Park Press, 1979:3-41.

7. Dominguez RH. Water polo injuries. In: Ciullo JV, editor. Clin Sports Med 1986;5:1.

8. Clarys JP, Cabri J, Teirlinck P. An electromyographic and impact fource study of the overhand water polo throw. In: MacLeran D, Reilly T, Less A, editors. Biomechanics and medicine in swimming - Swimming science VI. London: E \& FN Spon, Chapman \& Hall, 1992.
Apesar de algumas controvérsias no que diz respeito à predominância do sistema de energia durante os jogos de PA, parece que há uma certa tendência em aceitar a grande participação do sistema anaeróbio aláctico e aeróbio ${ }^{1,15,16}$.

\section{CONCLUSÕES}

Levando em consideração as limitações da magnitude da amostra e reprodutibilidade dos resultados encontrados, estes indicaram que não há a predominância do sistema glicolítico anaeróbio para o fornecimento energético durante o jogo de PA, o que conduz a hipótese de uma importante contribuição do sistema anaeróbio aláctico (ATP-CP), visto que há a realização de atividades de alta intensidade nos momentos decisivos do jogo e do metabolismo aeróbio no intervalo das ações.

É oportuna a elaboração de trabalhos subseqüentes controlando as ações dos jogadores através de imagens durante os jogos, além de um número maior de sujeitos envolvidos proporcionando assim informações mais conclusivas quanto à contribuição dos sistemas energéticos.

\section{AGRADECIMENTOS}

Aos profissionais: Bárbaro Diaz - Técnico da Seleção Brasileira Masculina de Pólo Aquático e Supervisor Técnico do Clube Internacional de Regatas/PMS/McDonalds. Antonio Edson Basaglia - Técnico do Clube Internacional de Regatas/PMS/McDonalds.

9. Davis T, Blanksby BA. A cinematographic analysis of the overhand water polo throw. J Sports Med Phys Fitness 1977;17:5-16.

10. Clarys JP. Biomechanical and morphological aspects of water polo. In: Wood GA, editor: Collected papers on sports biomechanics. Nedlands (WA): University of Western Australia, 1983:102-32.

11. Pinnington H, Dawson B, Blanksby B. The energy requirements for water polo. Nedlands (WA): Australian Sports Commission, 1986.

12. Sardella F, Alippi B, Rudic R. Analisi fisiometabolica della partita. Técnica Nuoto, 1990: 21-4.

13. Hohmann A, Frase R. Analysis of swimming speed and energy metabolism in competition water polo games. Proceedings of the Federation Internationale de Natation Amateur (FINA), First World Water Polo Coaches seminar. Athens, Lausanne: FINA, 1991:208-13.

14. Hohmann A. Analysis of delayed training effects in the preparation of the west German water polo team for the 1988 Olympic Games. In: MacLaren D, Reilly T, Less A, editors. Biomechanics and medicine in swimming - Swimming science VI. London: E \& FN Spon, 1992.

15. Konstantaki M, Trowbridge EA, Swaine IL. The relationship between blood lactate and heart rate responses to swim bench exercise and women's competitive water polo. J Sports Sci 1998;16:251-6.

16. Hollander AP, Dupont SHJ, Volkerijk SM. Physiological strain during competitive water polo games and training. In: Miyashita M, Mutoh Y, Richardson AB, editors. Medicine and science in aquatic sports. Medicine Sports Science, Basel, Karger, 1994;39:178-85. 
17. Williams JR, Armstrong N, Kirby BJ. The $4 \mathrm{mM}$ blood lactate level as an index of exercise performance in 11-13 year old children. J Sports Sci 1990;8:139-47.

18. Williams JR, Armstrong N. The influence of age and sexual maturation on children's blood lactate responses to exercise. Pediatric Exercise Science 1991;3:111-20.

19. Williams JR, Armstrong N. Relationship of maximal lactate steady state to performance at fixed blood lactate reference values in children. Pediatric Exercise Science 1991;3:331-41.
20. Avlonitou E. Energy requirements and training considerations in competitive water polo games. Proceedings of the Federation Internationale de Natation Amateur (FINA). First World Water Polo seminar. Athens, Lausanne: FINA, 1991:139-50.

21. Rodriguez FA. Physiological testing of swimmers and water polo players in Spain. In: Miyashita M, Mutoh Y, Richardson AB, editors. Medicine and science in aquatic sports. Medicine Sports Science, Basel, Karger, 1994;39:172-7.

MOVATEC (meloxicam). Contra-Indicacões; Não deve ser utilizado em pacientes que tenham apresentado hipersensibilidade ao meloxicam ou aos excipientes da sua fórmula. Existe possibilidade de sensibilidade cruzada com o ácido acetilsalicílico e outros antinflamatórios não-esteróides. Não administrar a pacientes que tenham apresentado distúrbios como asma, pólipos nasais, edema de Quincke ou urticária após o uso de ácido acetilsalicílico ou outros antiinflamatórios não-esteróides. Não deve ser administrado em casos de úlcera péptica ativa, insuficiência hepática grave e insuficiência renal grave. Não usar o produto em crianças e adolescentes menores de 15 anos de idade. Precaucóes: Pacientes com antecedentes de afecções do trato digestivo superior ou sob tratamento com anticoagulantes. 0 tratamento com meloxicam deve ser interrompido se ocorrer úlcera péptica ou sangramento gastroduodenal. 0 mesmo procedimento deve ser seguido em pacientes que apresentaram sinais de reaçōes cutâneo-mucosas adversas. Os antiinflamatórios não-esteróides inibem a síntese das prostaglandinas renais envolvidas na manutenção da perfusão renal. Nos pacientes que apresentam diminuicăo do fluxo sangüíneo e do volume sangüíneo renal, a administração de antiinflamatórios não-esteróides pode precipitar uma descompensação renal que, no entanto, via de regra, retorna ao estágio do pré-tratamento com a interrupção da terapêutica. Este risco atinge principalmente pacientes desidratados, idosos e portadores de insuficiência cardíaca congestiva, cirrose hepática, síndrome nefrótica ou insuficiência renal ativa, e os pacientes sob tratamento com diuréticos ou que sofreram uma intervenção cirúrgica de grande porte, responsável por um estado de hipovolemia. Nestes pacientes, controlar cuidadosamente o volume urinário e a função renal ao iniciar o tratamento. Em casos raros, os antiinflamatórios não-esteróides podem provocar nefrite intersticial, glomerulonefrite, necrose medular renal ou síndrome nefrótica. Nos pacientes com insuficiência renal grave, sob tratamento com hemodiálise, a dose de meloxicam não deve exceder 7,5 mg ao dia. Nos pacientes com disfunção renal leve ou moderada (clearance de creatinina $>25 \mathrm{~m} / \mathrm{min}$ ), não há necessidade de redução da dose. De modo geral, a dose diária não deve exceder $15 \mathrm{mg}$. Da mesma forma que com outros antiinflamatórios näo-esteróides, observaram-se elevaçöes ocasionais das transaminases séricas ou de outros indicadores da função hepática. Na maioria dos casos, esses aumentos foram transitórios e discretos. Se as alterações forem significativas ou persistentes, interromper a administração de meloxicam e solicitar os exames apropriados. Em caso de cirrose hepática clinicamente estável, não há necessidade de redução da dose. A tolerabilidade ao produto é menor em pacientes idosos, debilitados ou desnutridos, que devem ser observados mais de perto. A prudência deve ser maior nos pacientes idosos, nos quais as funçōes renais, hepáticas e cardíacas estão alteradas mais freqüentemente. Näo existem estudos específicos relativos a efeitos sobre a habilidade de dirigir carros ou operar máquinas; entretanto, se ocorrerem reaçöes adversas como vertigem e sonolência, recomenda-se omitir tais atividades. Gravidez e Lactaçäo: Embora não se tenham observado efeitos teratogênicos nos estudos pré-clínicos, näo há dados sobre a utilização de meloxicam durante a gravidez e a lactação humanas. M eloxicam não deve, portanto, ser utilizado durante a gravidez e o período de lactação. Interaçöes Medicamentosas: Outros antiinflamatórios não-esteróides, incluindo salicilatos, anticoagulantes orais, ticlopidina, heparina parenteral, trombolíticos, antidiabéticos orais, lítio, metotrexato; pacientes em uso de DIU (possibilidade de diminuição da eficácia do dispositivo), diuréticos, anti-hipertensivos (betabloqueadores, inibidores da ECA, vasodilatadores, diuréticos), colestiramina. Os antiinflamatórios não-esteróides podem aumentar a nefrotoxicldade da ciclosporina. Administração concomitante de antiácidos, cimetidina, digoxina ou furosemida não revelou interaçōes farmacocinéticas significativas. Reaçöes Adversas: Acima de 1\% - dispepsia, náusea, vômitos, dor abdominal, constipação, flatulência, diarréia, anemia, pruridos, erupção cutânea, tontura, cefaléia, edemas. Entre 0,1 e 1\% - alteraçōes transitórias dos parâmetros de função hepática, alteraçōes do hemograma, estomatite, urticária, vertigem, zumbido, sonolência, elevação da pressäo arterial, palpitaç̧öes, rubor facial, alteraçōes dos parâmetros de função renal. Abaixo de 0,1\%. colite, perfuração gastrintestinal, fotossensibilidade, aparecimento de asma aguda. Superdose: Em caso de superdose devem-se tomar as medidas-padräo de esvaziamento gástrico e de suporte geral. Desconhece-se um antídoto específico para meloxicam. Demonstrou-se em estudo clínico que a colestiramina acelera a eliminação de meloxicam. Indicaçōes: Tratamento sintomático da artrite reumatóide, tratamento sintomático das osteoartroses dolorosas. Posologia: Artrite reumatóide - 15 mg uma vez ao dia. De acordo com a resposta terapêutica, a dose pode ser reduzida para $7,5 \mathrm{mg}$ uma vez ao dia. $\underline{\text { osteoartrite }} \cdot 7,5 \mathrm{mg}$ uma vez ao dia. Em pacientes com insuficiência renal grave sob tratamento por hemodiálise, a dose diária não deve exceder $7,5 \mathrm{mg}$. De modo geral, a dose diária não deve exceder $15 \mathrm{mg}$. Versão: M OVAT-R-0007271. MS - 1.0367.0102 